\title{
Prostate cancer risk and aggressiveness associated with the CYP1B1 4326C/G (Leu432Val) polymorphism: a meta-analysis of 2788 cases and 2968 controls
}

\author{
Jie Yang*, Dong-Liang Xu*, Qiang Lu, Zhi-Jian Han, Jun Tao, Pei Lu, Chao Wang, Xiao-Ke Di and Min Gu
}

To derive a precise estimation of the associations between the cytochrome P450 1B1 (CYP1B1) 4326C/G variants and prostate cancer (PCa) risk or aggressiveness, a meta-analysis was performed using all eligible published studies. Odds ratios (ORs) with $95 \%$ confidence intervals (Cls) were estimated to assess the association in seven literature studies with $\mathbf{2 7 8 8}$ cases and 2968 controls. In the overall analysis, no significant association was found between the CYP1B1 4326C/G polymorphism and PCa risk, but ethnicity subgroup analyses and a case-source analysis revealed significant associations. The $4326 \mathrm{G}$ allele showed a significant association with increased PCa risk in Asians ( $O R=1.52,95 \% \mathrm{Cl}: 1.20-1.92$ ), and significant associations were also observed in a heterozygote comparison ( $\mathrm{OR}=1.40,95 \% \mathrm{Cl}: 1.03-1.89)$, a homozygote comparison $(\mathrm{OR}=2.38,95 \% \mathrm{Cl}: 1.31-4.33)$ and in a dominant genetic model $(\mathrm{OR}=1.52,95 \% \mathrm{Cl}: 1.14-2.01)$. Moreover, the $4326 \mathrm{G}$ allele was also significantly correlated with an increased risk of sporadic $\mathrm{PCa}(\mathrm{OR}=1.13,95 \% \mathrm{Cl}: 1.04-1.24)$, and significant associations were observed in a heterozygote comparison $(\mathrm{OR}=1.16,95 \% \mathrm{Cl}$ : $1.02-1.33)$, a homozygote comparison ( $\mathrm{OR}=1.24,95 \% \mathrm{Cl}: 1.03-1.49)$ and a dominant genetic model $(\mathrm{OR}=1.19,95 \% \mathrm{Cl}$ : $1.05-$ 1.34). The overall analyses and all subgroup analyses showed no significant association between the 4326C/G polymorphism and PCa aggressiveness. Our meta-analysis showed that CYP1B1 4326G allele is significantly associated with an increased PCa risk in Asians and in sporadic PCa cases.

Asian Journal of Andrology (2012) 14, 560-565; doi:10.1038/aja.2012.4; published online 16 April 2012

Keywords: aggressiveness; CYP1B1; meta-analysis; polymorphism; prostate cancer; risk

\section{INTRODUCTION}

Prostate cancer (PCa) is one of the most common solid tumours and a leading cause of death in men worldwide, but the incidence of PCa varies distinctively by ethnic group and geographic location. ${ }^{1,2}$ For example, there were approximately 192280 newly diagnosed cases and 27360 PCa-related deaths in the United States in 2008, and the incidence of PCa in African-Americans in the United States is approximately 60 times the incidence among the Han nationality population in China. ${ }^{1-3} \mathrm{PCa}$ can be divided into hereditary PCa (HPC) and sporadic PCa (SPC). HPC is confirmed when a patient has more than three first- or seconddegree relatives with $\mathrm{PCa}$, and patients not meeting these criteria are defined as having SPC. HPC is a heterogeneous disease with complex genetics. Although the onset of HPC is, on average, 6 years earlier than the SPC onset, the clinical courses are the same. ${ }^{1-3}$ To date, the underlying aetiology of PCa is still unknown, but a large number of studies have indicated that both genetic predispositions and environmental factors likely contribute to the risk and aggressiveness of $\mathrm{PCa}{ }^{4}$

The human cytochrome P450 1B1 (CYP1B1) gene is implicated in the activation of oestrogens and carcinogens and is differentially expressed, with high mRNA levels detected in prostate, mammary, kidney and other tissues. ${ }^{5-7}$ Furthermore, some studies have demonstrated that the expression of CYP1B1 and the formation of activated carcinogens are associated with several tumours, including PCa. ${ }^{6-8}$ Thus, we have reasons to believe that the tissue-specific hydroxylation and carcinogen activation initiated by CYP1B1 may affect PCa risk.

One mechanism leading to CYP1B1 overactivation is genetic polymorphism. Several polymorphic sites within the CYP1B1 gene (rs2617266, rs2567206, rs2551188, rs10012, rs1056836, rs1056827 and rs1800440) have been studied, but the rs1056836 (4326C/G, Leu432Val or L432V) site has been reported most frequently, albeit inconsistently, associated with increased PCa risk. ${ }^{9-15}$ Some researchers have investigated the relationship between the CYP1B1 4326C/G polymorphism and PCa aggressiveness, although the definitions of low and high aggressiveness are different among these research reports. ${ }^{9,10,12,14,15}$

We performed a meta-analysis of all eligible case-controlled studies to derive a more precise estimation of the associations between the CYP1B1 4326C/G variants and PCa risk or aggressiveness.

\section{MATERIALS AND METHODS}

Publication search

PubMed was searched with the terms 'CYP1B1', 'polymorphism' and 'prostate cancer' for articles published in English through 9 March 2011. All eligible studies were retrieved, and their bibliographies were checked for other relevant publications. Relevant review articles were 
hand-searched to identify additional eligible studies. If more than one article had been published using the same series of study subjects, only the most recent or most complete study for this meta-analysis were chosen.

\section{Inclusion and exclusion criteria}

The included articles had to be case-control studies, contain information on the association between the CYP1B1 4326C/G polymorphism and $\mathrm{PCa}$ risk or aggressiveness, and had to provide sufficient genotype frequencies for a meta-analysis. The most frequent reasons for study exclusion were the lack of a control population, the lack of sufficiently available data and study duplication.

\section{Data extraction}

All data were carefully and independently extracted from the eligible publications by two co-authors (Chao Wang and Xiao-Ke Di), and any disagreement was resolved by a discussion between the two authors. The collected data from each eligible article included the first author's last name, the year of publication, the country of origin, patient ethnicity, the author's definition of low- or high-tumour aggressiveness, the characteristics of the controls, the numbers of genotyped cases and controls, the numbers of genotyped cases of low and high aggressiveness, the source of the case groups (familial or sporadic prostate cancer cases), the genotyping methods and any quality controls. The patient ethnicities were categorised as Caucasian, Asian or African. When studies included subjects with both familial and sporadic $\mathrm{PCa}$, the genotype data were extracted separately for the subgroup analysis.

\section{Statistical analysis}

The strength of the association between the CYP1B1 4326C/G polymorphism and PCa risk or aggressiveness was measured by odds ratios (ORs) with 95\% confidence intervals (CIs). The statistical significance of the summary ORs were determined with $Z$-tests. To explore the association of the CYP1B1 4326C/G polymorphism and PCa risk, we analysed the allelotype comparisons (4326G vs. C) as well as the heterozygote comparisons (CG vs. CC), homozygote comparisons (GG vs. CC) and the dominant genetic model comparisons (CG+GG vs. $\mathrm{CC}$ ) between the cases and controls. For the association of the $4326 \mathrm{C} /$ $\mathrm{G}$ polymorphism with $\mathrm{PCa}$ aggressiveness, we evaluated these same effects between the low- and high-aggressiveness cases. Stratified analyses were performed by ethnicities and the case sources (i.e., HPC vs. $\mathrm{SPC})$.

The heterogeneity assumption was checked by a $\chi^{2}$-based $Q$-test. If the $Q$-test had a $P$ value greater than 0.05 , thereby indicating the lack of heterogeneity among the studies, the summary OR estimation of each study was calculated by the fixed-effects model (the
Mantel-Haenszel method). Otherwise, the random-effects model (the DerSimonian-Laird method) was adopted. ${ }^{16}$ Meta-regression analyses to explore the reasons for heterogeneity among these studies were performed. Subgroup analyses, which were grouped by characteristics including ethnicity and case source, were adopted to avoid the influence of heterogeneity on these studies. The inter-study variance $\left(I^{2}\right)$ was used to quantify the degree of heterogeneity, and the $I^{2}$ per cent was used to describe the extent of the heterogeneity explained by the subgroup characteristics. The potential publication bias was determined by an Egger's linear regression test with a funnel plot. All statistical analyses were performed with the Stata software (version 11.0; StataCorp LP, College Station, TX, USA) using two-sided $P$ values.

\section{RESULTS}

\section{The summary of included studies}

Seven literature studies that matched the inclusion criteria for our analysis of the association between PCa risk and the CYP1B1 4326C/ G polymorphism were retrieved. ${ }^{9-15}$ Of these, 5 studies were analysed for the association of the $4326 \mathrm{C} / \mathrm{G}$ polymorphism and $\mathrm{PCa}$ aggressiveness. ${ }^{9,10,12,14,15}$ The characteristics of each study are summarized in Table 1. All studies employed frequency-matched controls by age, sex and ethnicity. A PCR restriction fragment length polymorphism (PCR-RFLP) assay was adopted in four of the seven studies, and all studies mentioned genotyping quality control, including randomly repeated assays or validation by directed sequencing. The distribution of genotypes in the control groups was consistent with the HardyWeinberg equilibrium in all but two studies. ${ }^{11,14}$

Sobti et al. ${ }^{11}$ and Chang et al. ${ }^{13}$ studied only the association between $\mathrm{PCa}$ risk and the $4326 \mathrm{C} / \mathrm{G}$ polymorphism, and thus, these two studies were excluded when analysing the association between $\mathrm{PCa}$ aggressiveness and the $4326 \mathrm{C} / \mathrm{G}$ polymorphism. In addition, Chang et al. ${ }^{13}$ studied both sporadic and hereditary PCa risk with 103 HPC cases, 207 SPC cases and 182 common controls, and thus, the results of this study were divided into two different studies, each using the common control group, for the subgroup analysis (Table 2). Therefore, there were seven studies with 2788 cases and 2968 controls (containing twice the number of common controls for the study by Chang et al. ${ }^{13}$ ) for the PCa risk analysis and 5 studies with 1017 lowand 1167 high-aggressiveness cases for the PCa aggressiveness analysis. After grouping by ethnicity, there were two studies of Caucasian descendants, three of Asian descendants and three of a mixed group of descendants (more than $90 \%$ Caucasian) ${ }^{13,15}$ for the PCa risk analysis, and there were two studies of Caucasian descendants, two of Asian descendants and one of a mixed group of descendants (91\% Caucasian, $8 \%$ African American and 1\% Asian or Hispanic/ Latino $)^{15}$ for the PCa aggressiveness analysis. After grouping by case

Table 1 The main characteristics of included studies

\begin{tabular}{|c|c|c|c|c|c|c|c|c|c|}
\hline $\begin{array}{l}\text { First } \\
\text { author }\end{array}$ & Publication year & $\begin{array}{l}\text { Publish } \\
\text { country }\end{array}$ & $\begin{array}{l}\text { Subject } \\
\text { ethnicity }\end{array}$ & Case number & Case source & $\begin{array}{l}\text { Control } \\
\text { number }\end{array}$ & HWE test & $\begin{array}{l}\text { Genotyping } \\
\text { method }\end{array}$ & $\begin{array}{l}\text { Aggressiveness } \\
\text { analysis }\end{array}$ \\
\hline Cussenot $^{9}$ & 2007 & France & Caucasian & 1053 & SPC & 837 & Yes & PCR-TaqMan & Yes \\
\hline Beuten $^{10}$ & 2008 & USA & Caucasian & 633 & SPC & 733 & Yes & PCR-TaqMan & Yes \\
\hline Sobti ${ }^{11}$ & 2006 & India & Asian & 100 & SPC & 100 & NM & PCR-RFLP & NM \\
\hline Fukatsu ${ }^{12}$ & 2004 & Japan & Asian & 136 & SPC & 255 & Yes & PCR-RFLP & Yes \\
\hline Chang $^{13}$ & 2003 & USA & Mixed & 310 & $\mathrm{HPC} / \mathrm{SPC}$ & 182 & Yes & MassARRAY & NM \\
\hline Tanaka $^{14}$ & 2002 & Japan & Asian & 117 & SPC & 200 & NM & PCR-RFLP & Yes \\
\hline Cicek $^{15}$ & 2005 & USA & Mixed & 439 & HPC & 479 & Yes & PCR-RFLP & Yes \\
\hline
\end{tabular}

Abbreviations: HPC, hereditary prostate cancer; HWE, Hardy-Weinberg equilibrium; NM, not mentioned; PCR, polymerase chain reaction; RFLP, restriction fragment length polymorphism; SPC, sporadic prostate cancer. 
Table 2 Stratified analyses of CYP1B1 4326C/G polymorphism on Prostate Cancer (PCa) risk

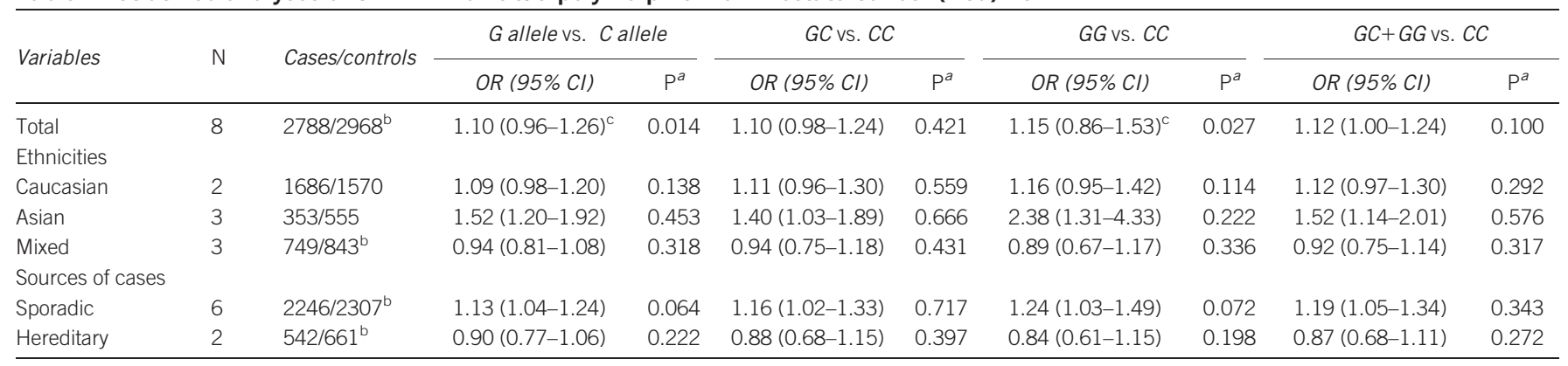

Abbreviations: $\mathrm{Cl}$, confidence interval; $N$, number of studies; $\mathrm{OR}$, odds ratio.

a $P$ value of $Q$-test for heterogeneity test.

${ }^{b}$ Numbers of the controls containing double the number of common controls for the studies by Chang et al. ${ }^{13}$

${ }^{\mathrm{c}} \mathrm{A}$ random-effects model was used when $P$ value for heterogeneity test was $<0.05$.

source, a total of six studies investigated SPC risk, two studies ${ }^{13,15}$ investigated HPC risk, four studies ${ }^{9,10,12,14}$ investigated SPC aggressiveness and one study ${ }^{15}$ investigated HPC aggressiveness.

\section{The CYP1B1 4326C/G polymorphism and PCa risk}

PCa risk is defined as the predicted likelihood of developing $\mathrm{PCa}$ during a man's lifetime. ${ }^{2}$ We observed a wide variation of CYP1B1 $4326 \mathrm{G}$ allele frequencies across different ethnicities. The frequency of the $4326 \mathrm{G}$ allele was $16.76 \%$ among Asian controls, which was significantly lower than in Caucasian controls $(38.66 \%, P<0.01)$ and in mixed controls $(46.22 \%, P<0.01)$. We also found that the frequency of the $4326 \mathrm{G}$ allele in hereditary cases was significantly higher than in sporadic cases $(44.19 \%$ vs. $38.58 \%, P<0.05)$, but there was no obvious difference between the frequencies of the $4326 \mathrm{G}$ allele between lowand high-aggressiveness cases $(39.63 \%$ vs. $39.46 \%, P>0.05)$.

In the overall analysis, we did not find any significant association between the CYP1B1 4326C/G polymorphism and PCa risk, but there was significant heterogeneity in the allelotype comparison $(P=0.014$ for heterogeneity) and the homozygote comparison $(P=0.027$ for heterogeneity). Therefore, we assessed the source of the allelotype heterogeneity by comparing the results by the publication year (before or after 1 January 2005), ethnicity, case source and sample size (more or less than 200 subjects in both cases and controls). The publication year $(P=0.796)$, ethnicity $(P=0.540)$, source of cases $(P=0.150)$ and the sample size $(P=0.375)$ were all unable to account for the substantial heterogeneity. In addition, the meta-regression analysis indicated that the source of cases could explain only $27.66 \%$ of the $I^{2}$.

We next performed subgroup analyses by stratifying by ethnicity or case source and revealed several interesting associations. First, the 4326G allele was significantly associated with an increased risk of $\mathrm{PCa}$ in Asians compared with the 4326C allele (OR $=1.52,95 \% \mathrm{CI}$ : $1.20-1.92, P=0.453$ for heterogeneity) (Figure 1). This association was further confirmed in the heterozygote comparison $(\mathrm{OR}=1.40$, 95\% CI: $1.03-1.89, P=0.666$ for heterogeneity), the homozygote comparison $(\mathrm{OR}=2.38,95 \% \mathrm{CI}: 1.31-4.33, P=0.222$ for heterogeneity) and in the dominant genetic model $(\mathrm{OR}=1.52,95 \% \mathrm{CI}: 1.14-2.01$, $P=0.576$ for heterogeneity) (Table 2). In contrast, no association was found between the polymorphism and PCa risk in either the Caucasian or mixed subject subgroup. Subdivision by case source also indicated that the $4326 \mathrm{G}$ allele was significantly correlated with increased PCa risk in sporadic cases $(\mathrm{OR}=1.13,95 \% \mathrm{CI}: 1.04-1.24$, $P=0.064$ for heterogeneity) (Figure 1), and similar results were observed in the heterozygote comparison with the 4326CC genotype ( $\mathrm{OR}=1.16,95 \% \mathrm{CI}: 1.02-1.33, P=0.717$ for heterogeneity), in the homozygote comparison $(\mathrm{OR}=1.24,95 \% \mathrm{CI}: 1.03-1.49, P=0.072$ for heterogeneity) and in the dominant genetic model $(\mathrm{OR}=1.19$, 95\% CI: 1.05-1.34, $P=0.343$ for heterogeneity) (Table 2). However, there was no significant correlation between the $4326 \mathrm{C} / \mathrm{G}$ polymorphism and HPC risk.

\section{CYP1B1 4326C/G polymorphism and PCa aggressiveness}

Five studies have investigated the relationship between the CYP1B1 4326C/G polymorphism and PCa aggressiveness, but the definitions of low and high aggressiveness vary among these research reports. ${ }^{9,10,12,14,15}$ Some articles define aggressiveness by the Gleason score, the clinical stage, and the preoperative prostate-specific antigen level or by a combination of these separate criteria. Therefore, for the meta-analysis of PCa aggressiveness, we defined low-aggressive potential as a Gleason score between 2 and 6 , a clinical stage of T1-T2-N0-M0 or a preoperative prostate-specific antigen level of less than $30 \mathrm{ng} \mathrm{ml}^{-1}$, and we defined high-aggressive potential as a Gleason score between 7 and 10, a clinical stage of $\mathrm{T} 3-\mathrm{T} 4$ or $\mathrm{N}+$ or $\mathrm{M}+$, or a preoperative prostatespecific antigen level of $30 \mathrm{ng} \mathrm{ml}^{-1}$ or higher.

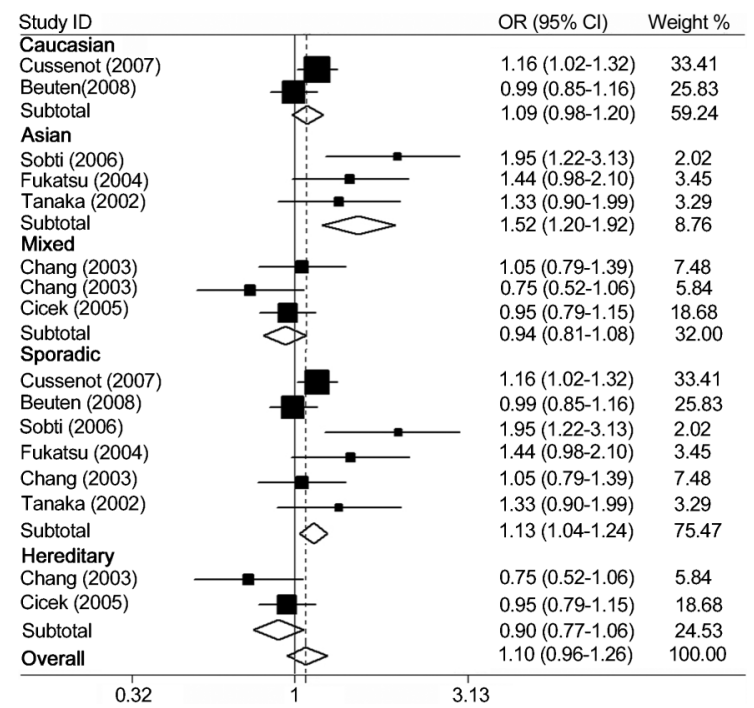

Figure 1 The forest plot of PCa risk associated with the CYP1B1 4326C/G polymorphism ( $G$ allele vs. C allele). The squares and horizontal lines correspond to the study-specific OR and $95 \% \mathrm{Cl}$. The area of the squares reflects the weight. The diamonds represent the summary $\mathrm{OR}$ and $95 \% \mathrm{Cl}$. $\mathrm{Cl}$, confidence interval; $\mathrm{OR}$, odds ratio; PCa, prostate cancer. 
In the overall analysis, there was no significant association between the $4326 \mathrm{C} / \mathrm{G}$ polymorphism and PCa aggressiveness (Figure 2), but we found significant heterogeneity in the allele comparison $(P=0.011$ for heterogeneity), in the homozygote comparison ( $P=0.029$ for heterogeneity) and in the dominant genetic model comparison $(P=0.024$ for heterogeneity) (Table 3). Therefore, we assessed the source of the heterogeneity in the allelotype comparison by stratifying by the publication year (before or after 1 January 2005), ethnicity, case source and sample size (more or less than 200 subjects in both the low- and high-aggressiveness cases or not). However, none of these concomitant variables could account for the substantial heterogeneity or the $I^{2}$ of the allelotype comparison by the meta-regression analyses. Additional subgroup analyses with stratification by ethnicity or case source also produced no significant association (Table 3 and Figure 2).

\section{Sensitivity analyses}

In the allelotype comparison for the association between $4326 \mathrm{C} / \mathrm{G}$ variants and PCa risk, our sensitivity analyses indicated that no single study influenced the summary OR or the 95\% CI significantly. Although two studies ${ }^{11,14}$ failed to meet the Hardy-Weinberg equilibrium, the summary OR and the 95\% CI were effectively unaltered by the inclusion of the two studies (Figure 3a). Similarly, the sensitivity analyses also demonstrated that no single study qualitatively influenced the summary OR or the $95 \% \mathrm{CI}$ in the allelotype comparison for the association of the $4326 \mathrm{C} / \mathrm{G}$ polymorphism and $\mathrm{PCa}$ aggressiveness (Figure 3b).

\section{Publication bias}

We adopted the Begg's funnel plot and Egger'ss test to assess the publication bias. The shape of the funnel plots appeared symmetric and did not suggest the presence of publication bias in either the allelotype comparison for PCa risk (Figure 3c) or in aggressiveness (Figure 3d). Egger's test was used to provide statistical evidence for the funnel plot symmetry. As expected, the results failed to indicate any obvious evidence of publication bias in both allelotype comparisons for PCa risk $(P=0.457)$ or aggressiveness $(P=0.539)$.

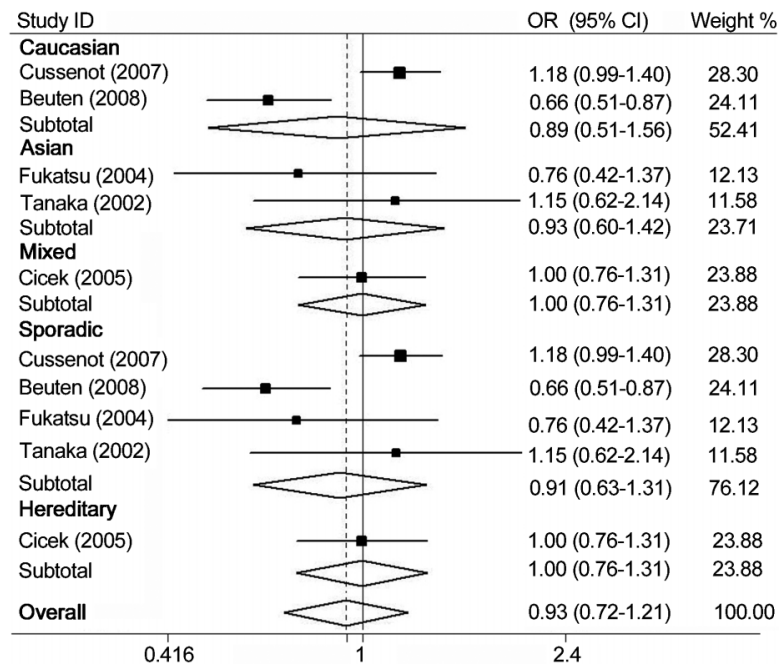

Figure 2 The forest plot of PCa aggressiveness associated with the CYP1B1 4326C/G polymorphism ( $G$ allele vs. $C$ allele). The squares and horizontal lines correspond to the study-specific OR and $95 \% \mathrm{Cl}$. The area of the squares reflects the weight. The diamonds represent the summary OR and $95 \% \mathrm{Cl}$. Cl, confidence interval; OR, odds ratio; $\mathrm{PCa}$, prostate cancer.

\section{DISCUSSION}

Prostate carcinogenesis is a multistep process involving multifactorial interplay between genetic and environmental factors. ${ }^{17}$ As a result, a single polymorphism study is unlikely to have any substantial effect and may be of limited value in predicting risk and aggressiveness. ${ }^{18}$ Therefore, to explore the association between CYP1B1 4326C/G variants and PCa risk or aggressiveness, we performed a meta-analysis including 2788 cases and 2968 controls from seven published studies. To the best of our knowledge, this is the first meta-analysis exploring the CYP1B1 4326C/G polymorphism in $\mathrm{PCa}$ risk and potential aggressiveness.

CYP1B1 is a member of the cytochrome P450 (CYP450) gene family and one of the major enzymes involved in oestrogen hydroxylation, ${ }^{18}$ a key reaction in hormonal carcinogenesis. ${ }^{19}$ Therefore, CYP1B1 is commonly implicated in hormone-mediated tumours, such as prostate, breast, endometrial, and ovarian cancer. CYB1B1 is expressed at particularly high levels in these cancers and is responsible for hormone metabolism and the formation of toxic metabolites from both endogenous and exogenous molecules. ${ }^{18,19}$ Interestingly, there have been reports that, in contrast to its overexpression in PCa tissue, CYP1B1 protein expression is not detected in normal prostate tissue. ${ }^{20,21}$ The importance of CYP1B1 in chemical carcinogenesis is also well illustrated in animal models, in which metabolites of CYP1B1 have been shown to induce PCa. ${ }^{5,22}$

Several family-based and case-controlled studies have demonstrated that individuals with the hyperactive 4326GG genotype are at higher risk for breast cancer. ${ }^{23-25}$ Other studies have indicated that the 4326GG genotype may also be associated with endometrial cancer risk in premenopausal women. ${ }^{26}$ However, the ovarian cancer risks associated with the CYP1B1 4326C/G polymorphism are not consistent in different races. Goodman et al. ${ }^{27}$ have found that the $4326 \mathrm{GG}$ and 4326GC genotypes are associated with a higher risk in the Hawaiian population (consisting of Asians and Caucasians), but a recent study by Cecchin et al. ${ }^{28}$ reported no difference between 4326GG cases and controls in Caucasian subjects.

In our study, the analysis indicated that the $4326 \mathrm{C} / \mathrm{G}$ polymorphism was not significantly associated with an overall PCa risk, but we found significant heterogeneity in the allelotype comparison and the homozygote comparison. We performed subgroup analyses, and the $4326 \mathrm{G}$ allele was found to be significantly associated with an increased PCa risk in Asians and sporadic cancer subjects in the allelotype comparison. The increased PCa risk in the Asian population was also associated with the GC genotype in the heterozygote comparison, the GG genotype in the homozygote comparison and the GC+GG genotypes in the dominant genetic model. No association was observed in Caucasians, mixed population subjects or in hereditary cases in all genetic models (Table 2). We believe that these inconsistent results may have several causes. First, it may result from the distribution of allele frequencies among different ethnicities or between different case sources. We found that the frequency of the $4326 \mathrm{G}$ allele was significantly lower in Asian controls than in Caucasian or mixed population controls (both $P<0.01$ ), and the frequency of the $4326 \mathrm{G}$ allele was significantly higher in hereditary cancer cases than in sporadic cases $(P<0.05)$. This finding is consistent with the higher incidence of $\mathrm{PCa}$ in Caucasians and Africans than in Asians and with the 2- to 11-fold increase in the lifetime risk of PCa in patients with a positive family history. ${ }^{1-4,19}$ Second, the mechanism of PCa development is similar to other cancers and is dependent on the interactions of genetic factors and environmental agents. ${ }^{29,30}$ Environments and lifestyles are very different among individuals of different races. ${ }^{31}$ In addition, 
Table 3 Stratified analyses of CYP1B1 4326C/G polymorphism on Prostate Cancer (PCa) aggressiveness

\begin{tabular}{|c|c|c|c|c|c|c|c|c|c|c|}
\hline \multirow{2}{*}{ Variables } & \multirow{2}{*}{$\mathrm{N}$} & \multirow{2}{*}{$\begin{array}{l}\text { High-/Low- } \\
\text { aggressiveness }\end{array}$} & \multicolumn{2}{|c|}{$G$ allele vs. $C$ allele } & \multicolumn{2}{|l|}{$G C$ vs. $C C$} & \multicolumn{2}{|l|}{$G G$ vs. $C C$} & \multicolumn{2}{|c|}{$G C+G G$ vs. $C C$} \\
\hline & & & OR $(95 \% \mathrm{Cl})$ & $\mathrm{P}^{a}$ & OR $(95 \% \mathrm{Cl})$ & $\mathrm{P}^{a}$ & OR $(95 \% \mathrm{Cl})$ & $\mathrm{P}^{a}$ & OR $(95 \% \mathrm{Cl})$ & $\mathrm{p}^{a}$ \\
\hline Total & 5 & $1017 / 1167$ & $0.93(0.72-1.21)^{\mathrm{b}}$ & 0.011 & $1.09(0.90-1.31)$ & 0.068 & $0.88(0.53-1.45)^{\mathrm{b}}$ & 0.029 & $0.98(0.70-1.37)^{\mathrm{b}}$ & 0.024 \\
\hline Caucasian & 2 & 720/802 & $0.89(0.51-1.56)^{\mathrm{b}}$ & 0.001 & $0.96(0.57-1.63)^{b}$ & 0.029 & $0.79(0.26-2.38)^{\mathrm{b}}$ & 0.001 & $0.91(0.46-1.78)^{\mathrm{b}}$ & 0.003 \\
\hline Asian & 2 & $109 / 140$ & $0.93(0.60-1.42)$ & 0.336 & $0.87(0.50-1.51)$ & 0.267 & $1.00(0.35-2.82)$ & 0.761 & $0.89(0.53-1.49)$ & 0.274 \\
\hline Mixed & 1 & $188 / 225$ & $1.00(0.76-1.31)$ & - & $1.50(0.96-2.35)$ & - & $0.93(0.56-1.56)$ & - & $1.26(0.84-1.89)$ & - \\
\hline Hereditary & 1 & $188 / 225$ & $1.00(0.76-1.31)$ & - & $1.50(0.96-2.35)$ & - & $0.93(0.56-1.56)$ & - & $1.26(0.84-1.89)$ & - \\
\hline
\end{tabular}

Abbreviations: $\mathrm{Cl}$, confidence interval; $N$, number of studies; $\mathrm{OR}$, odds ratio.

a $P$ value of $Q$-test for heterogeneity test.

${ }^{\mathrm{b}} \mathrm{A}$ random-effects model was used when $P$ value for heterogeneity test was $<0.05$.

individuals with a PCa family history may be more likely to pursue regular prostate exams, leading to an increased detection rate of $\mathrm{PCa}$ among these patients. Thus, the aetiology of PCa is unlikely to be explained solely by genetic polymorphisms because hereditary variation alone cannot affect the risk of any disease. ${ }^{20,21}$ Third, the sample sizes of the cases and controls in the Asian populations were both noticeably smaller than those in Caucasians (353/555 vs. 1686/1570) or mixed population subjects (353/555 vs. $749 / 843)$, and thus, the results of the Asian subject subgroup analysis may have a weaker statistical power and be at higher risk for sampling error and false positive results. In conclusion, further investigations with larger sample sizes are needed to validate these results. While other factors, such as time-lag bias and publication bias, might also be expected to play a role in biasing the study, we examined these possibilities and found no significant results.

To analyse the relationship between the $4326 \mathrm{C} / \mathrm{G}$ polymorphism and PCa aggressiveness, we investigated five studies with 1017 lowand 1167 high-aggressiveness cases. There was not any significant association in the overall analysis, and we found no obvious difference in the frequency of the $4326 \mathrm{G}$ allele between low- and high-aggressiveness cases $(P>0.05)$. Although we found significant heterogeneity for the allelotype, homozygote and dominant genetic model comparisons, subgroup analyses also did not reveal any significant associations. To assess the source of heterogeneity, we performed metaregression analyses for several concomitant variables, including the publication year, ethnicity, case source and the sample size, but none of these could account for the substantial heterogeneity or the $I^{2}$ of the allelotype comparison. We believe that the most probable source of heterogeneity is the difference in the definitions of low- and highaggressiveness $\mathrm{PCa}$, which could lead to the lack of any detected association and indicate that more studies with uniform definitions are needed to investigate the association between the $4326 \mathrm{C} / \mathrm{G}$ polymorphism and PCa aggressiveness.

Several limitations of this meta-analysis should be acknowledged. First, there was no independent study in Africans in the meta-analysis, which hindered the comprehensive investigation of the association
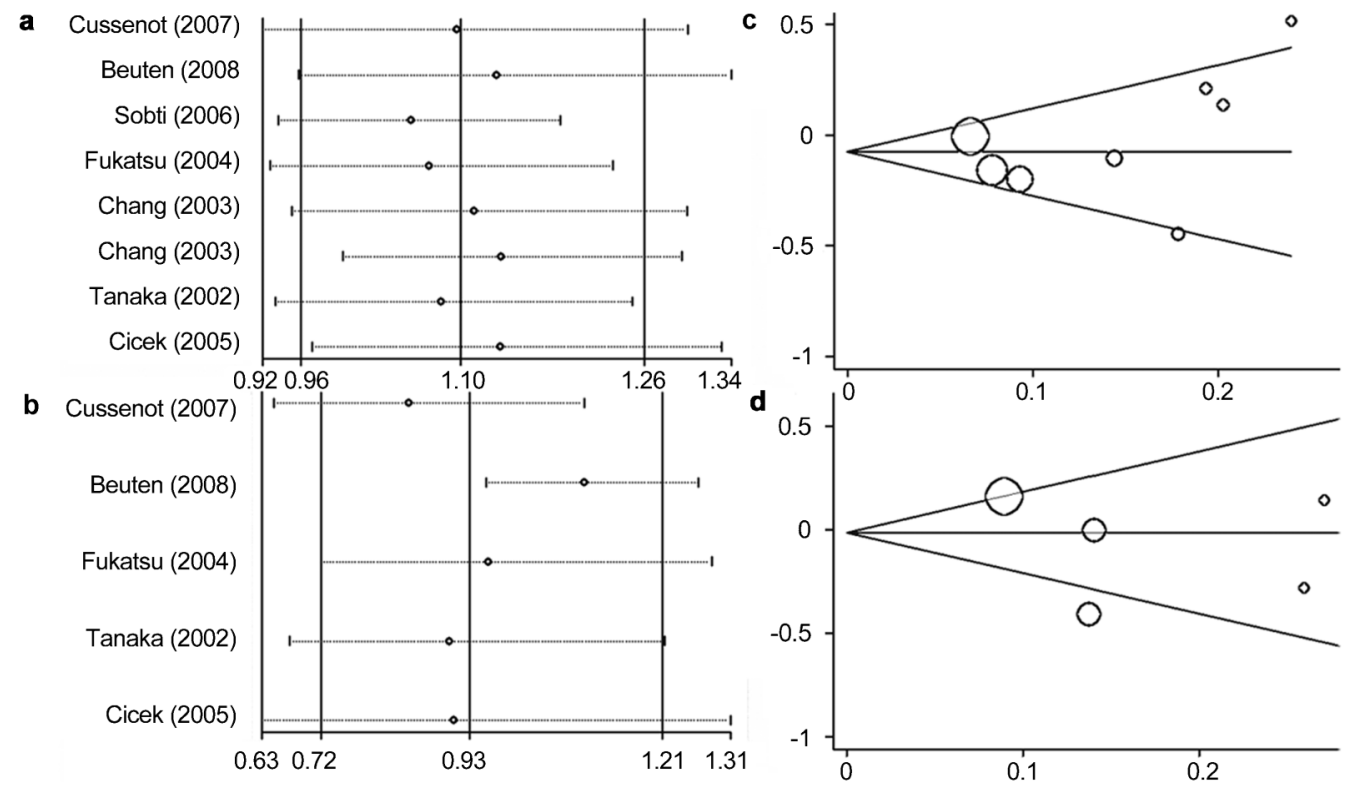

Figure 3 Sensitivity analysis pictures of each included study and Begg's funnel plots of the publication bias test for PCa risk and aggressiveness associated with the CYP1B1 4326C/G polymorphism (G allele vs. C allele). (a) Sensitivity analysis picture for the PCa risk and with the omission of the named study. (b) Begg's funnel plot for the publication bias test. Each point represents a separate study for PCa risk. (c) Sensitivity analysis picture for PCa aggressiveness. (d) Begg's funnel plot of the publication bias test for PCa aggressiveness. PCa, prostate cancer. 
between the CYP1B1 4326C/G polymorphism and PCa risk or aggressiveness. Second, the sample sizes of the cases and controls in Asians were relatively small, so the statistical power of these results was weaker than the power in Caucasians or mixed population subjects. We need further studies with larger sample sizes to confirm the positive associations in Asians. Third, PCa is considered the result of the combined effects of many factors, including inherited and environmental factors. However, our results were only based on unadjusted estimates that disregard the effect of environmental factors in tumorigenesis. ${ }^{29,30}$

Our meta-analysis also had some advantages. First, the aggressiveness of PCa was taken into account when analysing the association of the $4326 \mathrm{C} / \mathrm{G}$ polymorphism and PCa risk. Second, the quality of the studies included in our meta-analysis was satisfactory and met our strict inclusion criteria. Third, although two studies did not meet the Hardy-Weinberg equilibrium criterion, the sensitivity analyses indicated that no single study influenced the summary OR or the $95 \% \mathrm{CI}$ significantly. Fourth, we adopted Begg's funnel plot and Egger's test to assess the publication bias, and the results failed to show any obvious evidence of publication bias either for the PCa risk or aggressiveness.

\section{CONCLUSIONS}

Our meta-analysis demonstrated that the CYP1B1 4326G allele was significantly associated with an increased PCa risk in Asians and in sporadic PCa, but there was no obvious association between the 4326C/G polymorphism and PCa aggressiveness. Considering the limitations of the present meta-analysis, further research with standardised unbiased methods and larger, worldwide sample sizes are expected to confirm our results. Moreover, other possible confounding factors, including environmental factors and lifestyles, should be controlled and be taken into account when we assess the effect of inherited factors on PCa tumorigenesis.

\section{AUTHOR CONTRIBUTIONS}

JY, MG and DLX conceived and designed the study. CW, XKD and PL collected the data. QL, ZJH and JT performed the statistical analyses. JY and DLX drafted and revised the manuscript. All authors read and approved the final version.

\section{COMPETING FINANCIAL INTERESTS}

The authors declare that they have no competing financial interests.

1 Bono AV. The global state of prostate cancer: epidemiology and screening in the second millennium. BJU Int 2004; 94 Suppl 3, 1-2.

2 Zhang L, Yang BX, Zhang HT, Wang JG, Wang HL et al. Prostate cancer: an emerging threat to the health of aging men in Asia. Asian J Androl 2011; 13: 574-8.

3 Jemal A, Siegel R, Ward E, Hao Y, Xu J et al. Cancer statistics, 2009. CA Cancer J Clin 2009; 59: 225-49.

4 Schaid DJ. The complex genetic epidemiology of prostate cancer. Hum Mol Genet 2004; 13 Spec No. 1: R103-21.

5 Williams JA, Martin FL, Muir GH, Hewer A, Grover PL et al. Metabolic activation of carcinogens and expression of various cytochromes $\mathrm{P} 450$ in human prostate tissue. Carcinogenesis 2000; 21: 1683-9.

6 Yang X, Solomon S, Fraser LR, Trombino AF, Liu D et al. Constitutive regulation of CYP1B1 by the aryl hydrocarbon receptor (AhR) in pre-malignant and malignant mammary tissue. J Cell Biochem 2008; 104: 402-17.
7 Roos PH, Bolt HM. Cytochrome P450 interactions in human cancers: new aspects considering CYP1B1. Expert Opin Drug Metab Toxicol 2005; 1: 187-202.

8 Nebert DW, Dalton TP. The role of cytochrome P450 enzymes in endogenous signalling pathways and environmental carcinogenesis. Nat Rev Cancer 2006; 6 947-60.

9 Cussenot O, Azzouzi AR, Nicolaiew N, Fromont G, Mangin P et al. Combination of polymorphisms from genes related to estrogen metabolism and risk of prostate cancers: the hidden face of estrogens. J Clin Oncol 2007; 25: 3596-602.

10 Beuten J, Gelfond JA, Byrne JJ, Balic I, Crandall AC et al. CYP1B1 variants are associated with prostate cancer in non-Hispanic and Hispanic Caucasians. Carcinogenesis 2008; 29: 1751-7.

11 Sobti RC, Onsory K, Al-Badran Al, Kaur P, Watanabe M et al. CYP17, SRD5A2, CYP1B1, and CYP2D6 gene polymorphisms with prostate cancer risk in North Indian population. DNA Cell Biol 2006; 25: 287-94.

12 Fukatsu T, Hirokawa Y, Araki T, Hioki T, Murata T et al. Genetic polymorphisms of hormone-related genes and prostate cancer risk in the Japanese population. Anticancer Res 2004; 24: 2431-7.

13 Chang BL, Zheng SL, Isaacs SD, Turner A, Hawkins GA et al. Polymorphisms in the CYP1B1 gene are associated with increased risk of prostate cancer. Br J Cancer2003, 89: 1524-9.

14 Tanaka Y, Sasaki M, Kaneuchi M, Shiina H, Igawa M et al. Polymorphisms of the CYP1B1 gene have higher risk for prostate cancer. Biochem Biophys Res Commun 2002; 296: 820-6.

15 Cicek MS, Liu X, Casey G, Witte JS. Role of androgen metabolism genes CYP1B1, PSA $K L K 3$, and CYP1 1alpha in prostate cancer risk and aggressiveness. Cancer Epidemio Biomarkers Prev 2005; 14: 2173-7.

16 Moher D, Liberati A, Tetzlaff J, Altman DG, PRISMA GroupPreferred reporting items for systematic reviews and meta-analyses: the PRISMA statement. Ann Intern Med 2009; 151: 264-9.

17 Muller RL, Moreira DM. Smoking and prostate cancer survival and recurrence. Asian J Androl 2011; 13: 787-8.

18 Singh PB, Ragavan N, Ashton KM, Basu P, Nadeem SM et al. Quantified gene expression levels for phase I/II metabolizing enzyme and estrogen receptor levels in benign prostate from cohorts designated as high-risk (UK) versus low-risk (India) for adenocarcinoma at this organ site: a preliminary study. Asian J Androl 2010; 12 203-14.

19 Aprikian AG, Bazinet M, Plante M, Meshref A, Trudel C et al. Family history and the risk of prostatic carcinoma in a high risk group of urological patients. J Urol 1995; 154: 404-6.

20 Agundez JA. Cytochrome P450 gene polymorphism and cancer. Curr Drug Metab 2004; 5: 211-24.

21 Carnell DM, Smith RE, Daley FM, Barber PR, Hoskin PJ et al. Target validation of cytochrome P450 CYP1B1 in prostate carcinoma with protein expression in associated hyperplastic and premalignant tissue. Int J Radiat Oncol Biol Phys 2004; 58: 500-9.

22 Cavalieri EL, Devanesan P, Bosland MC, Badawi AF, Rogan EG. Catechol estrogen metabolites and conjugates in different regions of the prostate of Noble rats treated with 4-hydroxyestradiol: implications for estrogen-induced initiation of prostate cancer. Carcinogenesis 2002; 23: 329-33.

23 Listgarten J, Damaraju S, Poulin B, Cook L, Dufour J et al. Predictive models for breast cancer susceptibility from multiple single nucleotide polymorphisms. Clin Cancer Res 2004: 10: 2725-37.

24 Kocabas NA, Sardas S, Cholerton S, Daly AK, Karakaya AE. Cytochrome P450 CYP1B1 and catechol $O$-methyltransferase (COMT) genetic polymorphisms and breast cancer susceptibility in a Turkish population. Arch Toxicol 2002; 76: 643-9.

25 Sasaki M, Tanaka Y, Kaneuchi M, Sakuragi N, Dahiya R. Alleles of polymorphic sites that correspond to hyperactive variants of CYP1B1 protein are significantly less frequent in Japanese as compared to American and German populations. Hum Mutat 2003; 21: 652.

26 McGrath M, Hankinson SE, Arbeitman L, Colditz GA, Hunter DJ et al. Cytochrome P450 1B1 and catechol-O-methyltransferase polymorphisms and endometrial cancer susceptibility. Carcinogenesis 2004; 25: 559-65.

27 Goodman MT, McDuffie K, Kolonel LN, Terada K, Donlon TA et al. Case-control study of ovarian cancer and polymorphisms in genes involved in catecholestrogen formation and metabolism. Cancer Epidemiol Biomarkers Prev 2001; 10: 209-16.

28 Cecchin E, Russo A, Campagnutta E, Martella L, Toffoli G. Lack of association of CYP1 $B 1 * 3$ polymorphism and ovarian cancer in a Caucasian population. Int J Biol Markers 2004; 19: 160-3.29.

29 Bai JL, Zheng MH, Xia X, Ter-Minassian M, Chen YP et al MTHFR C677T polymorphism contributes to prostate cancer risk among Caucasians: a metaanalysis of 3511 cases and 2762 controls. Eur J Cancer 2009; 45: 1443-9.

30 Pfeifer GP. Environmental exposures and mutational patterns of cancer genomes. Genome Med 2010; 2: 54.

31 Sharad S, Srivastava A, Ravulapalli S, Parker P, Chen Y et al. Prostate cancer gene expression signature of patients with high body mass index. Prostate Cancer Prostatic Dis 2011; 14: 22-9. 